\title{
Line Graph Associated to Graph of a Near-Ring with Respect to an Ideal
}

\author{
Moytri Sarmah and Kuntala Patra
}

\begin{abstract}
Let $N$ be a near-ring and $I$ be an ideal of $N$. The graph of $N$ with respect to $I$ is a graph with $V(N)$ as vertex set and any two distinct vertices $x$ and $y$ are adjacent if and only if $x N y \subseteq I$ or $y N x \subseteq I$. This graph is denoted by $G_{I}(N)$. We define the line graph of $G_{I}(N)$ as a graph with each edge of $G_{I}(N)$ as vertex and any two distinct vertices are adjacent if and only if their corresponding edges share a common vertex in the graph $G_{I}(N)$. We denote this graph by $L\left(G_{I}(N)\right)$. We have discussed the diameter, girth, clique number, dominating set of $L\left(G_{I}(N)\right)$. We have also found conditions for the graph $L\left(G_{I}(N)\right)$ to be a cycle graph.
\end{abstract}

\section{Introduction}

Let $N$ be a right near-ring. Let $I$ be an ideal of $N$. A graph was defined by S. Bhavanari et.al.on a near-ring $N$ with respect to an ideal $I$ of $N$, denoted by $G_{I}(N) . G_{I}(N)$ is defined by considering all the elements of $N$ as vertices and any two distinct vertices are adjacent if and only if $x N y \subseteq I$ or $y N x \subseteq I$.

In this paper we define the line graph of $G_{I}(N)$, denoted by $L\left(G_{I}(N)\right) . L\left(G_{I}(N)\right)$ is the graph where vertex set is represented by all the edges of $G_{I}(N)$ and any two distinct vertices are adjacent if and only if their corresponding edges share a common vertex in the graph $G_{I}(N)$. If $x, y$ be two vertices adjacent in $G_{I}(N)$, then the corresponding vertex in the line graph $L\left(G_{I}(N)\right)$ is denoted by $[x, y]$.

A near-ring $N$ is called integral if it has no nonzero zero-divisors. $N$ is called simple if its ideals are $\{0\}$ and $N$. An ideal $I$ of $N$ is called prime, if for ideals $A, B$ of $N, A B \subseteq I$ implies either $A \subseteq I$ or $B \subseteq I$. An ideal $I$ is called semiprime if for any ideal $J$ of $N, J^{2} \subseteq I$ implies that $J \subseteq I$. An ideal $I$ is called 3-prime if for $a, b \in N$ and $a N b \subseteq I$ either $a \in I$ or $b \in I$. $N$ is called 3-prime near-ring if $\{0\}$ is a 3-prime ideal of $N$.

Let $G$ be a graph. The graph $G$ is said to be connected if there is a path between any two distinct vertices of $G$. On the other side, the graph $G$ is called totally disconnected if no two 
vertices of $G$ are adjacent. For vertices $x$ and $y$ of $G$, the distance between $x$ and $y$ denoted by $d(x, y)$ is defined as the length of the shortest path from $x$ to $y ; d(x, y)=\infty$, if there is no such path. The diameter of $G$ is $\operatorname{diam}(G)=\sup \{d(x, y): x, y$ are vertices of $G\}$. The girth of $G$, denoted by $\operatorname{gr}(G)$, is the length of a shortest cycle in $G ; \operatorname{gr}(G)=\infty$ if $G$ contains no cycle. A graph is said to be a cycle graph if it consists of a single cycle.

A dominating set for a graph $G$ is a subset $\mathrm{D}$ of the vertex set such that every vertex not in D is adjacent to at least one member of D.In a graph $G$ the maximal complete subgraph is called a clique. The number of vertices in a clique is called the clique number, denoted by $\omega(G)$.

For usual graph-theoretic terms and definitions, one can look at [1]. General references for the algebraic part of this paper are $[3,4,5,6]$.

Example 1. Let us consider $\mathbb{Z}_{4}$ the ring of integers modulo 4 . The ideals of $\mathbb{Z}_{4}$ are $I=\{0\}, J=$ $\{0,2\}$ and $K=\mathbb{Z}_{4}$. The graphs of $\mathbb{Z}_{4}$ with respect to ideals $I, J$ and $K$ and their corresponding line graphs are shown below in Figure 1 and Figure 2 respectively.
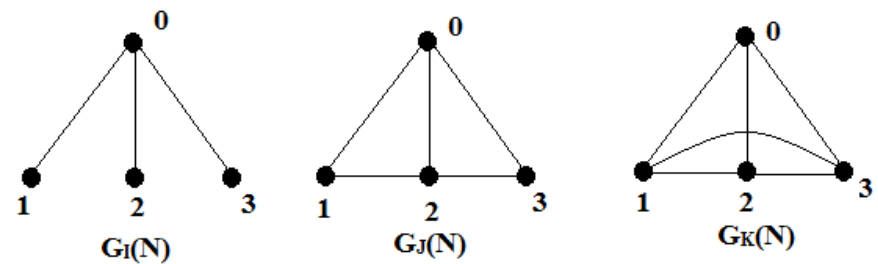

Figure 1: The graphs of $N=\mathbb{Z}_{4}$ with respect to ideals $I, J$ and $K$.

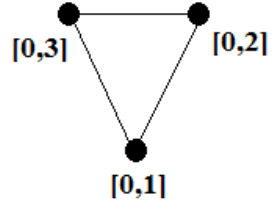

$\mathbf{L}\left(\mathrm{G}_{\mathbf{I}}(\mathrm{N})\right)$

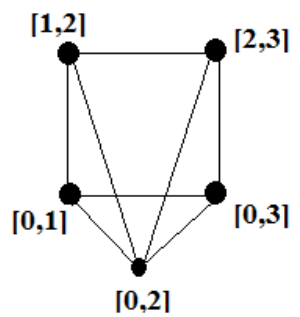

$\mathbf{L}(\mathbf{G J}(\mathbf{N}))$

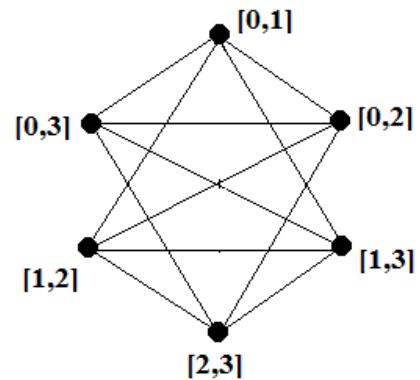

L(GK(N))

Figure 2: The Line graphs of $N=\mathbb{Z}_{4}$ with respect to ideals $I, J$ and $K$. 
Example 2. Let us consider a near-ring $N=\{0, a, b, c\}$ under the two binary operations ${ }^{\prime}+{ }^{\prime}$ and '. defined in the following tables:

\begin{tabular}{|c|c|c|c|c|}
\hline+ & 0 & $\mathrm{a}$ & $\mathrm{b}$ & $\mathrm{c}$ \\
\hline 0 & 0 & $\mathrm{a}$ & $\mathrm{b}$ & $\mathrm{c}$ \\
\hline $\mathrm{a}$ & $\mathrm{a}$ & 0 & $\mathrm{c}$ & $\mathrm{b}$ \\
\hline $\mathrm{b}$ & $\mathrm{b}$ & $\mathrm{c}$ & 0 & $\mathrm{a}$ \\
\hline $\mathrm{c}$ & $\mathrm{c}$ & $\mathrm{b}$ & $\mathrm{a}$ & 0 \\
\hline
\end{tabular}

\begin{tabular}{|c|c|c|c|c|}
\hline$\cdot$ & 0 & $\mathrm{a}$ & $\mathrm{b}$ & $\mathrm{c}$ \\
\hline 0 & 0 & 0 & 0 & 0 \\
\hline $\mathrm{a}$ & 0 & $\mathrm{a}$ & 0 & $\mathrm{a}$ \\
\hline $\mathrm{b}$ & $\mathrm{b}$ & $\mathrm{b}$ & $\mathrm{b}$ & $\mathrm{b}$ \\
\hline $\mathrm{c}$ & $\mathrm{b}$ & $\mathrm{c}$ & $\mathrm{b}$ & $\mathrm{c}$ \\
\hline
\end{tabular}

Here the ideals of $N$ are $I=\{0\}, J=\{0, b\}, K=\{0, b\}$ and $P=N$. The graphs of $N$ with respect to these ideals and their corresponding line graphs are shown below in Figure 3 and Figure 4 respectively.
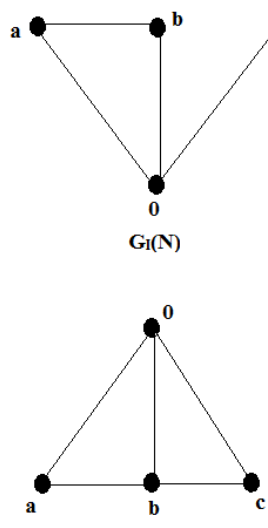

$\mathbf{G}_{\mathrm{K}}(\mathbf{N})$

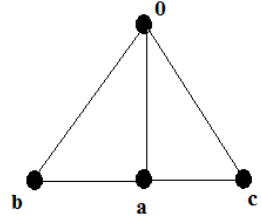

GJ(N)

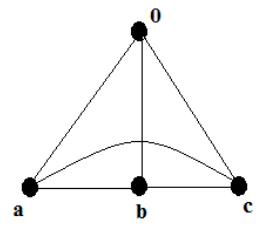

Gr(N)

Figure 3: The graphs of $N=\{0, a, b, c\}$ with respect to ideals $I, J, K$ and $P$.

Remark 1. Every ideal is itself a near-ring and so every ideal of a near-ing $N$ is a subnear-ring of $N$.

Theorem 1.1 ([2]). Let $N$ be integral as well as simple. Let I be an ideal of $N$. Then $G_{I}(N)=K_{n}$ or $G_{I}(N)$ is a rooted tree with root vertex 0 . 


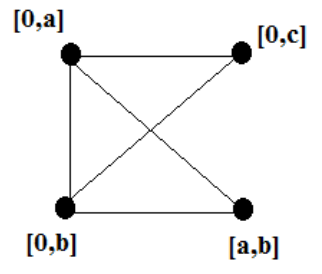

$\mathbf{L}\left(\mathbf{G}_{\mathbf{I}}(\mathbf{N})\right)$

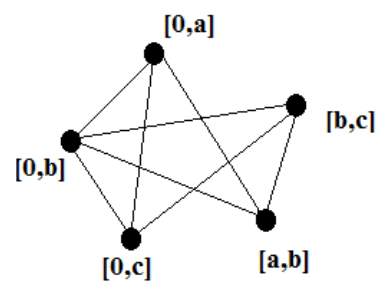

$\mathbf{L}\left(\mathbf{G K}_{\mathrm{K}}(\mathbf{N})\right)$

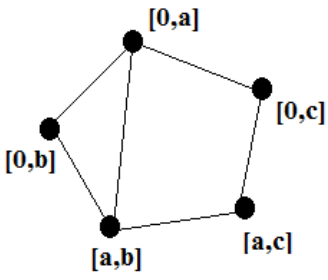

$\mathbf{L}(\mathbf{G} \mathbf{J}(\mathbf{N}))$

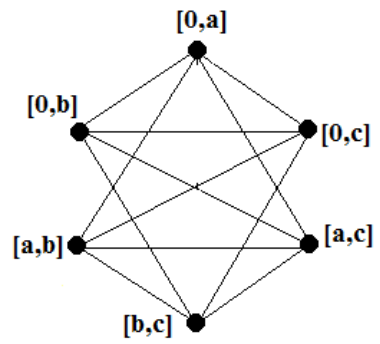

$\mathbf{L}(\mathbf{G P}(\mathbf{N}))$

Figure 4: The Line graphs of $N=\{0, a, b, c\}$ with respect to ideals $I, J, K$ and $P$.

\section{Main Results}

In this section we present some main results.Let $N$ be a right-near ring with $|N|=n$, where $|N|$ denotes the cardinality of $N, n$ may be of infinite cardinality also.

We begin our section with the following observation.

Remark 2. : In the graph $G_{I}(N), 0$ is always adjacent to all the other vertices resulting at least one edge and as a result its corresponding line graph will contain at least one vertex. Hence $L\left(G_{I}(N)\right)$ can never be an empty graph.

Theorem 2.1. For any near-ring $N$, the graph $L\left(G_{I}(N)\right)$ is always connected and $\operatorname{diam}\left(L\left(G_{I}(N)\right)\right) \leq$ 3.

Proof. In $G_{I}(N)$ the vertex 0 is adjacent to each vertex, so $G_{I}(N)$ is always connected and hence its line graph $L\left(G_{I}(N)\right)$ is always a connected graph.

Let us now prove the second part. Let $[x, y],[z, w]$ be any two vertices in $L\left(G_{I}(N)\right)$. Then we can always construct a path of length 3 as $[x, y]-[y, 0]-[0, z]-[z, w]$. Thus $\operatorname{diam}\left(L\left(G_{I}(N)\right)\right) \leq$ 3 .

Remark 3. If $N$ is simple and integral, then it is clear from Theorem 1.1. that the line graph $L\left(G_{I}(N)\right)$ is either a regular graph of degree $2 n-4$ or a complete graph $K_{n-1}$. 
The following results give the girth of $L\left(G_{I}(N)\right)$ under various conditions.

Theorem 2.2. For any near ring $N$ let $I=\{0\}$. Then $\operatorname{gr}\left(L\left(G_{I}(N)\right)\right)=\infty$ if and only if $N \cong \mathbb{Z}_{2}$ or $N \cong \mathbb{Z}_{3}$.

Proof. Let $N \cong \mathbb{Z}_{2}$. Then $G_{\{0\}}(N)$ is an edge $0-1$. Thus $L\left(G_{I}(N)\right)$ contains only one vertex and thus $\operatorname{gr}\left(L\left(G_{I}(N)\right)\right)=\infty$. Again let $N \cong \mathbb{Z}_{3}$. In this case $G_{\{0\}}(N)$ is a path of length 2, $1-0-2$ and so $\operatorname{gr}\left(L\left(G_{I}(N)\right)\right)=\infty$.

Conversely let $\operatorname{gr}\left(L\left(G_{I}(N)\right)\right)=\infty$. If possible let $N$ contains more than 3 elements. Suppose $|N|=4$. If $I=\{0\}$,then in $G_{I}(N)$ we will get an induced subgraph which is a star graph $K_{1,3}$. Thus the corresponding line graph will contain a 4 -cycle, which is a contradiction. Hence $N \cong \mathbb{Z}_{2}$ or $N \cong \mathbb{Z}_{3}$.

Theorem 2.3. For any near-ring $N$ if $G_{I}(N)$ contains a cycle, then $\operatorname{gr}\left(L\left(G_{I}(N)\right)\right)=3$,

Proof. If $G_{I}(N)$ contains a 3 cycle, then we are done.

Let $G_{I}(N)$ contains a 4 cycle. Thus $N$ contains atleast 4 elements. Since 0 is adjacent to every other vertex in $G_{I}(N)$, so there will be a star graph $K_{1,3}$ with centre vertex 0 . Hence the corresponding line graph will contain a subgraph isomorphic to $K_{3}$. Thus $L\left(G_{I}(N)\right)$ will contain a 3 -cycle and so $\operatorname{gr}\left(L\left(G_{I}(N)\right)\right)=3$.

Next let $G_{I}(N)$ contains a 5 -cycle. Then by the similar arguments given above $G_{I}(N)$ contains a star graph isomorphic to $K_{1,4}$. Hence in the corresponding line graph we will get a subgraph isomorphic to $K_{4}$. Therefore $\operatorname{gr}\left(L\left(G_{I}(N)\right)\right)=3$.

By The similar arguments if $G_{I}(N)$ contains a $n$ cycle, $n$ is any non zero positive integer, in $L\left(G_{I}(N)\right)$ there will be a complete subgraph isomorphic to $K_{n-1}$. Thus $L\left(G_{I}(N)\right)$ contains a 3 cycle. Hence $\operatorname{gr}\left(L\left(G_{I}(N)\right)\right)=3$. Thus the theorem is proved.

Remark 4. The converse of the Theorem 2.3. does not hold always. For example the line graph $L\left(G_{I}(N)\right)$ in Figure 2 has girth 3, but its corresponding graph $G_{I}(N)$ in Figure 1 does not contain any cycle.

Theorem 2.4. The line graph $L\left(G_{I}(N)\right)$ is a cycle graph if and only if either $N=I \cong \mathbb{Z}_{3}$ or $N \cong \mathbb{Z}_{4}$ and $I=\{0\}$.

Proof. Suppose $N=I \cong \mathbb{Z}_{3}$. It is clear from the definition that $G_{I}(N)$ is a cycle of length 3 and hence the corresponding line graph is a cycle graph that is $C_{3}$. Next suppose $N \cong \mathbb{Z}_{4}$ and $I=\{0\}$. In this case also $G_{I}(N)$ is a complete bipartite graph $K_{1,3}$ and it implies that the corresponding line graph is a cycle of length 3 .

Conversely let the line graph $L\left(G_{I}(N)\right)$ be a cycle graph. If possible let $N=I \nsubseteq \mathbb{Z}_{3}$. From the definition it is clear that $G_{I}(N)$ is a complete graph if $N=I$ that is $G_{I}(N)=K_{n}$. Therefore 
if $N=I \nsubseteq \mathbb{Z}_{3}$, then $G_{I}(N)=K_{n}, n \geq 4$. Hence the corresponding line graph contains more than one cycles, a contradiction. Again let $I=\{0\}$ and $N \nsubseteq \mathbb{Z}_{4}$. If $N \cong \mathbb{Z}_{3}$ or $\mathbb{Z}_{2}$, then the corresponding line graph does not contain any cycle, in fact the line graph is a path. Next, let $N \nsubseteq \mathbb{Z}_{n}, n \geq 5$. Thus in $G_{I}(N)$ we get a spanning subgraph $K_{1, n-1}$ which will induce a complete graph $K_{n-1}$ in $L\left(G_{I}(N)\right)$. Thus $L\left(G_{I}(N)\right)$ contains more than one cycle which is a contradiction. Thus the theorem is proved.

Next let us find the clique number in the graph $L\left(G_{I}(N)\right), \omega\left(L\left(G_{I}(N)\right)\right)$.

Theorem 2.5. For any ideal I of $N$, we have $\omega\left(L\left(G_{I}(N)\right)\right)=n-1$, where $n=|N|$.

Proof. Let $n=|N|$. For any ideal $I$ of $N$, in the graph $G_{I}(N), 0$ is adjacent to every other vertex inducing a spanning subgraph $K_{1, n-1}$. Thus in $L\left(G_{I}(N)\right), n-1$ vertices are adjacent to each other resulting a complete graph $K_{n-1}$. For suppose $L\left(G_{I}(N)\right)$ contains a subgraph isomorphic to $K_{n}$. Thus $n$ vertices are connected to each other and hence $n$ edges share a common vertex in $G_{I}(N)$. Therefore that common vertex has degree $n$ which is a contradiction since the maximum connectivity of any vertex in $G_{I}(N)$ is $n-1$. It implies that any other set having more than $n-1$ edges, the corresponding vertices in the line graph are not all adjacent to each other. Hence $\omega\left(L\left(G_{I}(N)\right)\right)=n-1$.

Theorem 2.6. For any near-ring $N$ if $G_{I}(N)$ does not contain a cycle then $L\left(G_{I}(N)\right)$ is a complete graph $K_{n-1}$, where $n=|N|$.

Proof. Let $G_{I}(N)$ contains no cycle. It implies that any two nonzero elements $x, y \in N$ are not adjacent in the graph $G_{I}(N)$, otherwise these elements together with 0 will form a cycle in $G_{I}(N)$ and hence same in $L\left(G_{I}(N)\right)$. Thus $G_{I}(N)$ is a star graph with 0 as a centre vertex and the corresponding line graph is a complete graph. Since In $G_{I}(N)$ there are $n-1$ edges, so $L\left(G_{I}(N)\right)$ is a complete graph with $n-1$ vertices that is $K_{n-1}$.

Theorem 2.7. For any ideal I of a near ring $N$, if $[x, y]$ is a vertex in $L\left(G_{I}(N)\right)$, then deg $([x, y])$ is atleast two.

Proof. Let $[x, y]$ be a vertex in $L\left(G_{I}(N)\right)$. Since 0 is adjacent to all other vertices in $G_{I}(N)$, thus there exist at least two edges $0-x$ and $0-y$ and as a result in the corresponding line graph we get two vertices $[0, x],[0, y]$. These two vertices are adjacent to $[x, y]$. Thus the result is proved.

Theorem 2.8. Let $I$ be a 3-prime ideal of $N$. Then the set $D=\{[x, n]: x \in I, n \in N\}$ is a dominating set in $L\left(G_{I}(N)\right)$. 
Proof. Let $I$ be a 3-prime ideal of the near-ring $N$. Then if $x-y$ is an edge in $G_{I}(N)$, it implies either $x N y \subseteq I$ or $y N x \subseteq I$. Without loss of generality, let $x N y \subseteq I$. since $I$ is a 3-prime ideal, so either $x$ or $y \in I$. let $x \in I$. Thus for any edge in $G_{I}(N)$ at least one end vertex of that edge will belong to $I$. It implies that $I$ is a dominating set for $G_{I}(N)$. Thus the edge set $\{(x-n): x \in I, n \in N\}$ will be an edge dominating set and thus $\{[x, n]: x \in I, n \in N\}$ is a vertex dominating set for the corresponding line graph $L\left(G_{I}(N)\right)$.

\section{References}

[1] F. Harary, Graph Theory, 1969 by Addison-Wesley Publishing Company, Inc.

[2] H. R. Maimani, M. R. Pournaki and S. Yassemi, Necessary and sufficient conditions for unit graphs to be hamiltonian, Pacific Journal of Mathematics, Vol. 249. No.2, 2011.

[3] K. Chowdhury, Near-rings and near-ring groups with finiteness conditions, VDM Verlag Dr.Muller Aktiengesellschaft and Co.KG, Germany, 2009.

[4] G. Pilz, Near-Rings, North-Holland Publishing Company, Amsterdam. New York. Oxford.1977.

[5] S. Bhavanari, S. P. Kuncham and B. S. Kedukodi, Graph of a nearring with respect to an ideal, Communication in Algebra, 38, (2010), 1957 - 1967.

[6] W. B. Vasantha Kandasamy, Smarandache near-rings, American Reasearch Press, Rehoboth, 2002.

Moytri Sarmah Department of Mathematicas, Girijananda Chowdhury Institute of Management and Technology, Guwahati-781017, India

E-mail: moytrisarmah@gmail.com

Kuntala Patra Department of Mathematics, Gauhati University, Guwahati-781014, India E-mail: kuntalapatra@gmail.com 